Revue de droit comparé du travail et de la sécurité sociale

3 | 2019

Les migrations internationales de travail

\title{
Migrations internationales et travail en Côte d'Ivoire
}

Nanga Silué

\section{(2) OpenEdition}

1 Journals

Édition électronique

URL : https://journals.openedition.org/rdctss/1468

DOI : $10.4000 /$ rdctss. 1468

ISSN : 2262-9815

Éditeur

Centre de droit comparé du travail et de la sécurité sociale

Édition imprimée

Date de publication : 1 novembre 2019

Pagination : 94-107

ISSN : 2117-4350

\section{Référence électronique}

Nanga Silué, "Migrations internationales et travail en Côte d'Ivoire ", Revue de droit comparé du travail et de la sécurité sociale [En ligne], 3 | 2019, mis en ligne le 01 novembre 2021, consulté le 13 novembre 2021. URL : http://journals.openedition.org/rdctss/1468 ; DOI : https://doi.org/10.4000/rdctss.1468

\section{cc)}

Revue de droit comparé du travail et de la sécurité sociale est mise à disposition selon les termes de la Licence Creative Commons Attribution - Pas d'Utilisation Commerciale - Pas de Modification 4.0 International. 


\title{
MigRATIONS INTERNATIONALES ET TRAVAIL EN CÔTE D'IVOIRE
}

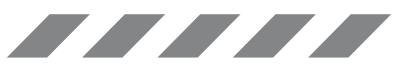

\begin{abstract}
Due to the situation of full employment that Côte d'Ivoire has experienced since colonial times and the policy of openness of President Félix Houphouët-Boigny, for whom what binds workers, namely humanity, comes before what distinguishes them, particularly their difference in nationality, the foreigner's work has never been a problem. It became one with the economic crisis that emerged in the 1970's.
\end{abstract}

KEYWORDS: Migration, Labour, Equality, Discrimination, Difference.

\section{RÉSUMÉ}

Grâce à la situation de plein emploi que la Côte d'Ivoire a connu depuis l'époque coloniale et la politique d'ouverture du Président Félix Houphouët-Boigny pour qui ce qui unit les travailleurs, à savoir I'humanité, passe devant ce qui les distingue, notamment leur différence de nationalité, le travail de l'étranger n'a jamais été un problème. Il en est devenu un avec la crise économique qui a fait son apparition dans les années 1970.

MOTS CLÉS: Migrations, travail, égalité, discrimination, différence. 
actualité ivoirienne donne à voir que le sujet sur les migrations internationales, avec pour fil d'Ariane le statut de l'étranger face au travail, renaît de ses cendres avec le débat sur «l'ivoirité »". Au moins, trois raisons expliquent ce sentiment de repli: la méconnaissance de l'étranger, la crainte du chômage ${ }^{2}$ et la prétendue insécurité.

Le tableau ainsi présenté correspond-il à celui de l'étranger? De quel étranger parlet-on exactement? Ces questions sont importantes car si le Code du travail emploie ce vocable, il ne le définit pas. C'est la loi sur l'entrée et le séjour des étrangers en Côte $\mathrm{d}^{\prime}$ Ivoire à laquelle renvoie le Code du travail ${ }^{3}$ qui précise cette notion. L'article $1^{\text {er }}$ de cette loi dispose ainsi: "Sont considérés comme étrangers au sens de la présente loi tous individus qui n'ont pas la nationalité ivoirienne, soit qu'ils aient une nationalité étrangère, soit qu'ils n'aient pas de nationalité ${ }^{4}$. Selon l'article $1^{\text {er }}$ de la Convention de 1954 relative au statut des apatrides, l'apatride est « une personne qu'aucun État ne considère comme son ressortissant en application de sa législation ». Sont donc étrangers, le non national et l'apatride. Sans utiliser le terme d'étranger, d'autres dispositions du Code du travail ${ }^{5}$ ainsi

1 Nous faisons allusion à la polémique actuelle entre le gouvernement qui a mis en garde le Président Henri Konan BEDIE contre ses propos de nature à susciter la haine des étrangers. En effet, alors qu'il recevait le 5 juin 2019 une délégation de son Parti, le PDCI RDA, l'ancien président BEDIE s'est saisi du conflit intercommunautaire du 20 mai 2019 à Béoumi entre les populations autochtones de l'ethnie baoulé et allogène de l'ethnie malinké (conflit qui a généré 11 morts, 108 blessés et 300 personnes déplacées) et des dégâts matériels importants, en affirmant: "Nous avons fait venir des étrangers dans nos plantations de café, de cacao; et ensuite, les gens se sont installés à leur propre compte, et aujourd'hui, ils agressent les planteurs ivoiriens et se disputent même la propriété des terres; il faut que nous réagissions pour que les Ivoiriens ne soient pas étrangers chez eux, car actuellement on fait en sorte que l'Ivoirien soit étranger chez lui. Mais les Ivoiriens n'accepteront jamais cela...».

2 N. Ferré, S. Nadal, "Traitement des travailleurs en France: regards critiques », in Mélanges A. Lyon-Caen, Dalloz, 2018, p. 348: "Le départ des uns, non autorisés à accéder au marché de l'emploi, résoudrait le problème des autres, frappés par le chômage ».

3 Loi n²015-532 du 20 juillet 2015 portant Code du travail, JORCl, n 74 du 14 octobre 2015, p. 1211 ; Article 26.8 alinéa 1er : « Les dispositions du présent chapitre (chapitre 6 du Code du travail relatif au voyage et transports du salarié de sa résidence habituelle jusque sur le lieu du travail et vice versa) ne peuvent être un obstacle à l'application de la réglementation sur les conditions d'admission et de séjour des étrangers ».

4 Loi n 90-437 du 29 mai 1990 relative à l'entrée et au séjour des étrangers en Côte d'Ivoire, JORCl, $\mathrm{n}^{\circ}$ du 9 août 1990, p. 262.

5 C'est le cas de l'article 2 du Code du travail qui fait de la nationalité un motif de discrimination. 
que des textes pris pour son application ${ }^{6}$ y font allusion. L'étranger auquel renvoie cette étude doit donc être distingué de l'usage travesti qui l'assimile à la personne de nationalité ivoirienne ayant quitté sa ville ou région natale pour s'installer dans une autre région de la Côte d'Ivoire ${ }^{7}$. Telle que présentée, l'étude présente un intérêt certain. Elle permet de poser la question de la migration professionnelle. La plus grande aspiration de l'étranger en terre d'accueil est de bénéficier d'une égalité de traitement avec les nationaux. Mais comment appréhender l'égalité de personnes qui n'ont pas la même nationalité? La différence de nationalité ne rend-elle pas impensable l'égalité de traitement? La Constitution n'est pas très claire dans la mesure où la nationalité n'a pas été retenue dans l'article $14^{8}$ au titre des motifs de discrimination dans l'emploi. La comparaison juridique 9 révèle que le sujet intéresse tous les États démocratiques soucieux du respect des droits fondamentaux de la personne, dans la mesure où, quel que soit le pays, l'étranger est souvent moins bien traité que le national. C'est pourquoi en période de crise, il est le premier à être victime de licenciement ${ }^{10}$. Or, en dépit de ce constat, la Côte d'Ivoire n'a pas ratifié la Convention de I'OIT sur les travailleurs migrants. Cette étude est l'occasion d'aborder toutes ces difficultés, aussi bien théoriques que pratiques.

La question fondamentale que l'on est en droit de se poser consiste à savoir, si en droit du travail, l'étranger est traité de la même façon que l'Ivoirien.

6 Voir notamment les articles 7 et 9 du décret $n^{\circ} 96-287$ du 3 avril 1996 relatif au contrat de travail, qui utilisent l'expression « travailleur d'une autre nationalité » en lieu et place d'étranger.

7 La précision est importante à cause de la fâcheuse tendance de certains Ivoiriens, se considérant "plus Ivoiriens que les autres », qui au lieu de s'en tenir au critère juridique de la nationalité pour arrêter leur conception de l'étranger, assimilent d'autres Ivoiriens - notamment les Malinkés - aux étrangers pour la seule raison qu'il existerait une synonymie entre leur nom patronymique et ceux des ressortissants des pays frontaliers tels que la Guinée, le Mali, le Burkina Faso. Or, cette conception de l'étranger est l'une des causes de la crise que la Côte d'Ivoire a connue et est en réalité à l'origine des tensions actuelles dans plusieurs régions de la Côte d'Ivoire.

8 Loi n 2016-886 du 8 novembre 2016 portant Constitution de la République de Côte d'Ivoire, JORCl, n 16, numéro spécial du 9 novembre 2016, p. 131 : «Toute personne a le droit de choisir librement sa profession ou son emploi. L'accès aux emplois publics et privés est égal pour tous en fonction des qualités et des compétences. Est interdite toute discrimination dans l'accès aux emplois ou dans leur exercice, fondée sur le sexe, l'ethnie ou les opinions politiques, religieuses ou philosophiques».

9 Voir par exemple dans les pays membres de l'Union européenne où la question est abordée depuis une trentaine d'année ; voir A. Lyon-Caen, "La libre circulation des travailleurs dans la Communauté économique européenne», Droit social, 1989, p. 526.

10 CSCJ, 22 mai 2014, arrêt $n^{\circ}$ 360/14 inédit. Pour mettre fin au contrat de travail d'un salarié de nationalité étrangère dans le cadre d'un licenciement pour motif économique, l'employeur, expliquant le licenciement litigieux, a affirmé: "Des postes particulièrement coûteux occupés présentement par un cadre expatrié verront leurs attributions confiées à des cadres locaux ». Face à une telle affirmation, le salarié de nationalité étrangère, suivi par les juges du fond, avait estimé avoir été victime de discrimination fondée sur la nationalité. Les juges du fond ne furent pas suivis par la Cour suprême. 
La réponse à cette question nécessite de remonter à l'histoire du salariat des étrangers en Côte d'Ivoire. Bien qu'ayant la même origine que les autres pays africains d'expression française, le droit du travail ivoirien n'a pas eu la même approche. Dotée de terres cultivables, la Côte d'Ivoire a été confrontée depuis l'époque coloniale ${ }^{11}$ à une insuffisance de maind'œuvre pour leur exploitation. Ce fut d'ailleurs la raison fondamentale du rattachement de la colonie de la Haute Volta (actuel Burkina Faso) à la colonie de Côte d'Ivoire entre 1937 et $1942^{12}$. Cette situation favorable à l'immigration des étrangers vers la Côte d'Ivoire a été encouragée par une politique très libérale adoptée par Félix Houphouët-Boigny, d'abord en tant que Député et Ministre de la France d'Outre-mer, ensuite en tant que Président de la République de Côte d'Ivoire en 1960.

Mais à l'époque coloniale, le terme d'étranger n'avait pas la même signification qu'aujourd'hui. Eu égard au destin commun des indigènes face au travail, pour Félix Houphouët-Boigny ${ }^{13}$ et ses compagnons de lutte ${ }^{14}$, l'étranger n'était pas le Malien, le Sénégalais ou le Béninois. Cette notion renvoyait au citoyen, français ou européen, qui n'avait pas le statut de droit local et par conséquent bénéficiait d'une différence de traitement enviable. L'abolition du travail forcé dont Houphouët-Boigny fut la cheville ouvrière ${ }^{15}$ et l'adoption du Code du travail des territoires d'Outre-mer de $1952^{16}$ figurent parmi les deux grandes victoires de ce combat pour l'égalité des travailleurs.

En prenant ainsi racine dans la lutte pour la décolonisation, l'égalité ici étudiée n'a jamais constitué un projet normatif autonome et a toujours été engluée dans la lutte générale pour l'égalité entre les personnes humaines. Pour F. Houphouët-Boigny, la nationalité n'est

11 K. Brou, Y. Charbit, «La politique migratoire de la Côte d'Ivoire », Revue européenne de migrations internationales, vol. 10, n 3, CERPAA CERPOD ORSTOM-Migrations africaines, 1994, p. 33.

12 Sur la suppression de la colonie de la Haute Volta, voir Décret du 5 septembre 1932 portant suppression de la Colonie de la Haute-Volta et répartition de son Territoire entre les Colonies du Niger, du Soudan français et de la Côte d'Ivoire, JOAOF, 15 octobre, p. 902. Sur le rétablissement de la colonie de la Haute Volta, voir Loi n ${ }^{\circ}$ 47-1707 du 4 septembre 1947 tendant au rétablissement du territoire de la Haute Volta, JOAOF, 27 septembre 1947. Voir également la "pétition des peuples voltaïques à Monsieur le Ministre de la France d'Outre-mer en vue de la renaissance de la Haute Volta dans les limites premières », in A. S. Balima, Genèse de la Haute Volta, Paris, Presses africaines, 1970, p. 164.

13 F. Houphouët-Boigny: «Alors accordez nous immédiatement l'égalité dans la législation du travail, dans le bénéfice de la sécurité sociale, l'égalité dans les allocations familiales, en bref l'égalité dans les niveaux de vie (...). Il faudrait pour atteindre ce but, que la totalité des Français consentent à un abaissement de 25 à 30 pour cent de leur niveau de vie au profit de nos compatriotes des territoires d'Outre-mer », cité par F. Cooper, «Empire, nation et citoyenneté : la France et l'Afrique »,

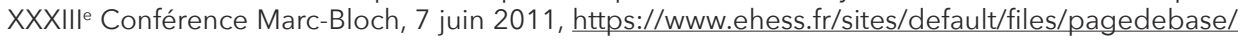
.../2011_conference_marc_bloch.pdf, p. 8.

14 "Les Africains comme toutes les populations de territoires d'Outre-mer, ont le plus vif sentiment d'appartenir à une collectivité, à un pays, à une "partie locale" du Sénégal, Soudan, Dahomey, Tchad, Gabon, etc., qui fait partie de la République française, mais qui se distingue des autres membres de la communauté française par son originalité géographique, historique et humaine, par ses conditions de vie économiques, psychologiques et religieuses ».

15 Loi n 46-645 du 11 avril 1946 tendant à la suppression du travail forcé dans les territoires d'Outremer.

16 Loi $n^{\circ}$ 52-1322 du 15 décembre 1952 portant Code du travail des territoires d'Outre-mer, JORF, $n^{\circ} 298$, p. 11541. 
pas le critère pertinent de définition des droits des étrangers, mais " l'humanité ${ }^{17}$. II a ainsi procédé au recrutement massif d'étrangers dans la Haute administration ${ }^{18}$ pour la construction de la nation ivoirienne.

Le droit du travail ivoirien porte l'empreinte de cette volonté initiale d'assimilation de l'étranger à l'Ivoirien. Le changement est intervenu avec la crise économique des années 1970. À cette époque, une politique défavorable à l'immigration du travail a été adoptée avec la création d'un Ministère dit de « l'ivoirisation des postes ${ }^{19}$. Plus tard, ce dispositif limitant l'emploi des étrangers a été enrichi par des dispositions réglementaires ${ }^{20}$ dont le législateur a fini par s'emparer en 2015, privilégiant ainsi « la préférence nationale » dans l'emploi ${ }^{21}$.

Dès l'origine, en raison de son histoire coloniale empreinte d'inégalité à l'égard des travailleurs indigènes, la Côte d'Ivoire a fait le choix de l'assimilation de l'étranger en lui promettant une égalité de traitement avec l'Ivoirien et les mêmes conditions de travail que lui (I). Mais avec la crise économique et face à son corolaire - la rareté de l'emploi-, l'étranger n'est désormais plus le bienvenu (II).

\section{I - L'ÉGALITÉ DE TRAITEMENT DE L'ÉTRANGER PROMISE}

Lorsque la volonté d'instaurer une égalité de traitement entre les salariés ivoiriens et étrangers a été clairement affichée, la question s'est posée de la formulation de cette égalité (A). Pour ne pas reproduire les erreurs du passé, fondées sur les différences de statuts, le législateur a mis en place certaines garanties afin que l'égalité proclamée ne soit pas illusoire (B).

\section{A - LA FORMULATION DE LA PROMESSE}

Dans sa formulation de l'égalité, le législateur a privilégié deux approches de l'égalité, inspirées des réflexions savantes d'Aristote ${ }^{22}$, à savoir l'indifférence à la différence de nationalité d'une part, et la prise en compte de la différence de nationalité lorsque celle-ci s'avère nécessaire d'autre part.

17 F. Houphouët-Boigny, cité par F. Cooper, op. cit., p. 23: « Ce que nous faisons en Côte d'Ivoire pour aider nos frères, c'est de les accueillir. Et nous les accueillerons avec un humanisme à l'africaine, empreint de fraternité vraie et de solidarité vraie ».

18 «Nous avons eu dans nos gouvernements des ministres venus de pays africains amis mais étrangers. Nous avons des préfets étrangers. Nous avons des ambassadeurs étrangers. Nous avons des chefs de services étrangers. Et nous avons dans la vie quotidienne, des paysans originaires d'autres territoires africains qui, avec les mêmes droits que les ivoiriens, participent à la construction nationale $"$.

19 Décret $n^{\circ} 78-875$ du 13 octobre 1978 portant organisation du Ministère du travail et de l'ivoirisation des cadres et portant réorganisation dudit ministère, JORCl du 9 novembre 1978, p. 2234.

20 Arrêté n6421 du 15 juin 2004 portant modification de l'arrêté n 1437 du 19 février 2004 règlementant le recrutement et les frais de visa du contrat de travail des personnels non-ivoiriens, JORCI n 29 du 15 juillet 2004, p. 241.

21 Voir article 11.1 de la loi $n^{\circ} 2015-532$ du 20 juillet 2015 portant Code du travail, $\mathrm{JORCl}^{\circ} \mathrm{n}^{\circ} 74$ du 14 juillet 2015, p. 1198, qui fait dépendre le recrutement de l'étranger de la preuve par l'employeur qu'il n'a pas trouver d'Ivoirien capable d'occuper le poste. Sur cette question, voir N. Yéo, «La préférence nationale en droit ivoirien du travail », Revue Ivoirienne des Sciences Juridiques et Politiques, 2016, n 4, p. 52.

22 Aristote, Éthique à Nicomaque, Paris, Flammarion, 1992, V, III, p. 142. 
L'indifférence à la différence de nationalité des salariés a commencé sous l'époque coloniale, avec l'avènement d'un droit du travail à visage humain. Le symbole en fut la loi du 15 décembre 1952 portant Code du travail dans les territoires d'Outre-mer. Cette loi a donné un sens aux principes de la liberté de travail - consacrée par la loi Houphouët-Boigny - et de l'égalité des personnes - reconnue par la Constitution française de 1946. Avant cette date, une distinction était faite entre le salarié indigène, notamment ivoirien, à qui était appliqué le droit colonial du travail différent du Code du travail français applicable aux Français et aux autres salariés européens. Cette situation avait pour fondement l'application du principe de la spécialité législative ${ }^{23}$.

L'indifférence à la différence de nationalité ne s'est pas limitée à cette pensée de l'égalité que $\mathrm{H}$. Kelsen range dans l'égalité devant la loi, définie à travers le principe de la régularité de l'application du droit en général ${ }^{24}$. Le législateur a exigé que le contenu de la loi ne fasse pas de distinction selon l'origine du salarié. La définition neutre du salarié ${ }^{25}$ par rapport au critère de la nationalité, l'alignement des droits du salarié étranger sur ceux du national - aussi bien dans les conditions de travail qu'au regard du principe « à travail égal, salaire égal $»^{26}$ - en sont des exemples éloquents.

En accédant à l'indépendance, au lieu de faire comme certains pays tels que le Mali qui, dès 1962, a limité l'accès au travail salarié des étrangers ${ }^{27}$, ce dispositif hérité de la colonisation et favorable à l'assimilation des étrangers aux Ivoiriens a été renforcé. En

23 Le principe de la spécialité législative consacre la différence de législation applicable dans les colonies et en France, qui rappelle que les colonies seront régies par des lois ou des règlements particuliers. La première formulation du principe de la spécialité législative résulte de l'article 91 de la Constitution du 22 Frimaire An VIII qui dispose que «Le régime des colonies françaises est déterminé par des lois spéciales ॥. II sera rappelé régulièrement par d'autres dispositions jusqu'à la veille de I'Indépendance en 1958 (article 73 de la Constitution française du 4 octobre 1958). Pour une idée exacte du principe de la spécialité législative, voir S. Boni, "Le droit de propriété foncière rurale en Côte d'Ivoire », thèse doctorat, Univ. F. Houphouët-Boigny, Abidjan, 2015, p. 31.

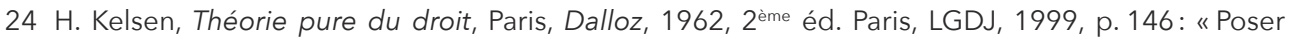
l'égalité devant la loi, c'est poser simplement que les organes d'application du droit n'ont le droit de prendre en considération que les distinctions qui sont faites dans les lois à appliquer ellesmêmes, ce qui revient à affirmer tout simplement le principe de la régularité de l'application du droit en général ».

25 Voir article 1 $1^{\text {er }}$ alinéa 1 ${ }^{\text {er }}$ : Est considéré comme travailleur ou salarié au sens de la présente loi, quels que soient son sexe et sa nationalité, toute personne qui s'est engagée à mettre son activité professionnelle, moyennant rémunération, sous la direction et l'autorité d'une autre personne, physique ou morale, publique ou privée. Pour la détermination de la qualité de travailleur, il ne sera tenu compte ni du statut juridique de l'employeur, ni de celui du salarié ».

26 Art. 91 du Code du travail des territoires d'Outre-mer: "À conditions égales de travail, de qualification professionnelle et de rendement, le salaire est égal pour tous les travailleurs quels que soient l'origine, le sexe, leur âge et leur statut dans les conditions prévues au présent titre ».

27 O.O. Sidibé, « Quel modèle d'inspiration pour le droit du travail malien depuis le Code du travail de 1952 », Bulletin de droit comparé du travail et de la sécurité sociale, Comptrasec, 2005, p. 144. Ainsi selon l'article 21 du Code du travail malien de 1962, "Les employeurs ne peuvent recruter les travailleurs étrangers qu'après avoir obtenu l'autorisation de la Direction Nationale de Travail dans les conditions qui seront déterminées par arrêté du Ministre chargé du Travail ». Par ailleurs, il précise que « Le chef de gouvernement pourra par décret pris en conseil de Ministres, en fonction des nécessités économiques, démographiques, sociales ou sanitaires, interdire ou limiter les possibilités d'embauche des entreprises, ou organiser les compensations en main d'œuvre entre les régions». 
témoignent la ratification de la Convention nº 111 de I'OIT dès $1961^{28}$ et l'adoption d'un Code du travail ${ }^{29}$ qui, à l'instar de la Convention de l'OIT, ont érigé la nationalité en motif de discrimination.

Mais dans la mesure où l'égalité de traitement signifie également nécessité de traiter différemment des personnes se trouvant dans des situations différentes, le législateur a isolé des situations dans lesquelles la différence de nationalité a un impact sur la relation de travail et donc rend incongrue une identité de traitement. Ce fut le cas des contrats d'expatriation. Une distinction a néanmoins été faite entre le contrat d'expatriation de droit commun et le contrat d'expatriation d'exception.

Le contrat d'expatriation qui emporte différence de traitement se présente sous deux formes.

La première a été consacrée par l'article 94 du Code du travail des territoires d'Outre$m e r^{30}$. De l'interprétation de ce texte, le contrat d'expatriation ne peut être conclu que par un salarié étranger. Le contentieux portant sur ce texte confirme cette interprétation ${ }^{31}$. C'est ainsi que l'on distinguait le contrat de travail de «type local», et le contrat de travail de «type expatrié $»^{32}$.

28 Voir art. 1er de la Convention $n^{\circ} 111$ de l'OIT: «1. Aux fins de la présente convention, le terme discrimination signifie : a) toute distinction, exclusion ou préférence fondée sur la race, la couleur, le sexe, la religion, I'opinion politique, l'ascendance nationale ou l'origine sociale qui a pour effet de détruire ou d'altérer l'égalité de chances ou de traitement en matière d'emploi et de profession ».

29 Loi n 64-290 du 1er août 1964 portant Code du travail, JORCl, n 44 spécial du 17 août 1964, p. 1059.

30 Article 94 alinéa $1^{\text {er }}$ du Code du travail des territoires d'Outre-mer: "Lorsque les conditions climatiques de la région du lieu d'emploi diffèrent de celles caractérisant la résidence habituelle d'un travailleur et lorsqu'il résultera pour ce dernier des sujétions particulières du faite de son éloignement du lieu de sa résidence habituelle au lieu d'emploi, le travailleur recevra une indemnité destinée à le dédommager des dépenses et risques supplémentaires auxquels l'exposent sa venue et son séjour au lieu d'emploi ».

31 CSCJ, 23 novembre 1962, Sieur Flandre (transporteur) c/sieur Mignot, inédit. Pour faire droit au jugement qui reconnait la qualité de travailleur expatrié à une personne de nationalité française, la Cour suprême a jugé que: "Attendu que la qualité d'expatrié donnant droit au congé sur la base de 5 jours par mois de service effectif, suivant les dispositions des articles combinés $95-3^{\circ}$ et $121 \mathrm{du}$ CTOM auxquels renvoie l'article 122 visé au moyen, ne dépend pas du lieu de recrutement du salarié mais de la situation de sa résidence habituelle et qu'aux termes de l'article 2 de l'arrêté $n^{\circ} 6556$ du 3/9/1952 dispositions transitoires pour l'attribution du congé en AOF, le critère retenu est la situation hors des limites de l'Afrique occidentale, qu'on ne saurait donc faire grief à la décision attaquée d'avoir, par une appréciation souveraine, admis que Flandre originaire de la France d'où il est venu en exécution d'un contrat de travail conclu avec le précédent employeur, avait la qualité d'expatrié ».

32 CSCJ, arrêt n 302/12 du 19 avril 2012, inédit: Un salarié de nationalité française titulaire d'un contrat de travail de "type expatrié » sollicite par un pourvoi le bénéfice d'une prime de bilan réservée au salarié titulaire de contrat de «type local». Le pourvoi est rejeté au motif que: "L'employé a été informé lors de son engagement que sa rémunération revue à la hausse tous les ans tient compte de la prime d'ancienneté et de la gratification, comme résulte des bulletins de paie produits; d'autre part, lié par un contrat d'expatrié et non local, il ne peut bénéficier de la prime de bilan ». Voir également, CSCJ, 13 du 18 avril 2013, arrêt n² 221, inédit. 
Plus tard, les partenaires sociaux, à travers l'article 57 de la Convention collective interprofessionnelle de 1977 intitulé «indemnité d'expatriation», se sont appropriés le contrat d'expatriation en l'enrichissant d'un aspect qui travestit l'esprit de départ. Ce texte consacre deux catégories de contrat d'expatriation. Le premier, aux alinéas 1 er et 2 , renvoie au contrat prévu par le Code du travail des territoires d'Outre-mer et conclu par un étranger ${ }^{33}$. Le second type, qui est en réalité une pathologie du contrat dit " d'expatrié », est conclu non pas au profit de l'étranger mais par un Ivoirien appelé à exécuter son contrat de travail hors de sa résidence habituelle située à au moins 500 kilomètres ou plus.

Hormis cette différence fondamentale, les raisons qui fondent la différence de traitement dans ces deux contrats dits d'expatrié sont quasi identiques, à savoir les sujétions particulières supportées par le salarié qui tiennent notamment aux conditions climatiques et à l'éloignement (qui nécessite un déplacement du lieu ou du pays de résidence au lieu d'emploi). Dans ces textes, la nature de la différence de traitement est également précisée. Il s'agit d'une indemnité qui vient compenser une perte financière subie du fait du travail. À ce titre, l'article 94 du Code des territoires d'Outre-mer parle de dédommagement pour les dépenses et risques supplémentaires auxquels la venue au lieu du travail expose le salarié étranger. Bien qu'ayant une origine ancienne, les contrats d'expatrié n'ont pas encore été contestés dans leur principe en droit ivoirien.

La seconde formule du contrat d'expatriation digne de la différence de traitement participant de la promesse d'égalité de traitement des travailleurs a été constatée dans une Convention signée en mars 1960 avec le Burkina Faso. Contrairement à I'hypothèse précédente, cette Convention relative à l'expatriation des travailleurs burkinabé en Côte d'Ivoire n'a pas évoqué la question de l'éloignement, encore moins celle du dépaysement. Elle a surtout visé certains points qui cristallisent généralement l'inégalité de traitement du salarié étranger, tels les conditions de travail, le salaire et le droit à une protection sociale.

Mais le législateur ivoirien avait compris, qu'il ne servait à rien de promettre l'égalité de traitement des étrangers dans l'emploi sans travailler concomitamment sur les conditions de de sa concrétisation, d'où un certain nombre de garanties prévues pour atteindre ce résultat.

\section{B - LES GARANTIES DE LA PROMESSE}

Les garanties de la promesse d'égalité se sont manifestées à deux niveaux: la recherche de la cohérence entre le droit de l'immigration et le droit à l'emploi de l'étranger, et l'intégration de ce dernier à la communauté nationale.

Concernant la première garantie, la nécessaire cohérence des droits évoqués a été relevée dans l'article 26.8 du Code du travail qui règle le conflit au profit du droit de l'immigration ${ }^{34}$. L'un des points fort de cette garantie a été de rendre possible l'accès au territoire ivoirien, condition permettant de faire partie du périmètre des égaux. Dans cette perspective, trois catégories d'étrangers ont été retenus. La première concerne les

33 Art. 57 de la Convention collective interprofessionnelle de 1977 fait allusion à «tout travailleur recruté hors du territoire de la République de Côte d'Ivoire et déplacé de sa résidence habituelle par le fait de l'employeur $"$.

34 Art. 26.8 alinéa $1^{\text {er }}$ de la loi nº 2015-15-532 du 20 juillet 2015 portant Code du travail, qui précise que les dispositions du Code du travail «ne peuvent être un obstacle à l'application de la réglementation sur les conditions d'admission et de séjour des étrangers ». 
ressortissants de l'Union Économique et Monétaire Ouest Africaine (UEMOA) ${ }^{35}$. La seconde vise les étrangers originaires de la Communauté Économique des États de l'Afrique de I'Ouest (CEDEAO) ${ }^{36}$ et la troisième est relative aux ressortissants d'États tiers. Pour les deux premières catégories, un principe fondamental gouverne le statut de l'étranger. II s'agit de la liberté de circulation des personnes. Tout citoyen de I'Union ${ }^{37}$ ou tout citoyen de la Communauté (CEDEAO) ${ }^{38}$, peut circuler librement et s'installer dans un autre État pour y exercer la profession de son choix. La seule différence entre ces deux statuts concerne les frais de visa, plus élevés lorsque l'étranger est citoyen de la Communauté.

Il importe cependant de relever que la liberté de circulation et d'accès au travail de l'étranger en Côte d'Ivoire n'a pas pour origine les traités de la CEDEAO et de I'UEMOA, la pratique ayant précédé le droit. En effet, jusqu'en 1990, date de l'adoption de la première loi ivoirienne sur l'entrée et le séjour des étrangers en Côte d'Ivoire, il n'y a jamais eu une véritable politique migratoire. En pratique, en raison du climat favorable à l'accueil des étrangers et de l'absence de contrôle des flux migratoires, tout étranger pouvait se rendre librement en Côte d'Ivoire, s'y installer pour exercer une activité salariée ou non. Cette situation a eu pour effet de rendre caduque le décret-loi du 12 juin 1932 relatif au séjour des français et étrangers en $\mathrm{AOF}^{39}$ adopté sous l'époque coloniale et abrogé seulement par la loi de 1990 relative à l'entrée et au séjour des étrangers en Côte d'Ivoire ${ }^{40}$.

35 L'Union Économique et Monétaire Ouest Africaine (UEMOA), créée par Traité signé entre les États parties le 10 janvier 1994 à Dakar (Sénégal), est entrée en vigueur le 1er août 1994 après ratification par tous les États signataires. Elle comprend huit pays: le Benin, le Burkina, la Côte d'Ivoire, la Guinée Bissau, le Mali, le Niger, le Sénégal et le Togo. Son siège est situé à Ouagadougou au Burkina Faso.

36 La Communauté des États de l'Afrique de l'Ouest a été créée par Traité signé entre les États parties en 1975 à Lagos au Nigéria. Initialement cantonné à l'économie, le champ du Traité s'est étendu depuis 1993 la coopération politique entre les États membres. La CEDEAO comprend quinze États membres (après le retrait de la Mauritanie en 2000): le Benin, le Burkina Faso, Capo Verde, la Côte d'Ivoire, la Gambie, le Ghana, la Guinée, la Guinée Bissau, le Libéria, le Mali, le Niger, le Nigéria, le Sénégal, la Sierra Leone, le Togo.

37 Article 91 du Traité de I'UEMOA: «1) Sous réserve des limitations justifiées par des motifs d'ordre public, de sécurité publique et de santé publique, les ressortissants d'un État membre bénéficient sur l'ensemble du territoire de I'Union de la liberté de circulation et de résidence qui implique: - l'abolition entre les ressortissants des États membres de toute discrimination fondée sur la nationalité, en ce qui concerne la recherche et l'exercice d'un emploi, à l'exception des emplois dans la Fonction Publique; - le droit de se déplacer et de séjourner sur le territoire de l'ensemble des États membres; - le droit de continuer à résider dans un État membre après y avoir exercé un emploi ».

38 Voir l'article 59 du Traité de la CEDEAO relatif à l'immigration: «1. Les citoyens de la Communauté ont le droit d'entrée, de résidence et d'établissement et les États Membres s'engagent à reconnaitre ces droits aux citoyens de la Communauté sur leurs territoires respectifs, conformément aux dispositions des protocoles y afférents. 2. Les États Membres s'engagent à prendre toutes les mesures appropriées en vue d'assurer aux citoyens de la Communauté, la pleine jouissance des droits visés au paragraphe 1 du présent article. 3. Les États Membres s'engagent à prendre, au niveau national, les dispositions nécessaires pour assurer l'application effective du présent article ».

39 Décret-loi du 12 juin 1932 relatif au séjour des français et étrangers en AOF, JOAOF.

40 Voir article 29 de la loi n 90-437 du 29 mai 1990 relative à l'entrée et au séjour des étrangers en Côte d'Ivoire, JORCl, 9 août 1990, p. 264: "Sont abrogées toutes les dispositions antérieures contraires à la présente loi, notamment le décret-loi du 12 juin 1932 règlementant les conditions d'admission et de séjours des étrangers en AOF ». 
Aussi, on peut affirmer sans risque de se tromper que la pratique ivoirienne du travail des étrangers était en avance sur les conventions portant sur le même objet conclues entre certains États et la Côte d'Ivoire. Contrairement à la pratique ivoirienne, ces conventions étaient forcement limitées à des domaines précis, telles par exemple des Conventions de $1977^{41}$ et $1995^{42}$ conclues entre la Côte d'Ivoire et la France.

Mais pour favoriser l'égalité de traitement de l'étranger, le législateur ne s'est pas contenté de lui garantir l'accès au territoire ivoirien. Il lui a également assuré l'intégration à la communauté ivoirienne, ce qui constitue une condition de l'épanouissement dans le travail. Cependant, quel que soit le moyen utilisé - discours politique, loi ou règlement l'intégration à la communauté ivoirienne a été assimilée à une accession à la citoyenneté ivoirienne. Le point de départ de cette comparaison est à rechercher dans les actions du Président Houphouët-Boigny; d'abord sous l'époque coloniale lors de laquelle il proposait en lieu et place de la « confédération ${ }^{43}$ défendue par Senghor la « nationalité africaine $~^{44}$, puis au lendemain de l'indépendance, lorsqu'il suggérait à ses pairs membres du Conseil de l'entente et de l'Assemblée nationale ivoirienne l'adoption du principe de la double nationalité. Alors même que la proposition fut rejetée en interne par l'Assemblée nationale ivoirienne, il a contourné ce refus en procédant au recrutement massif d'étrangers dans tous les secteurs d'activité, du secteur de l'éducation ${ }^{45}$ jusque dans la Haute administration ivoirienne ${ }^{46}$. Outre ces étrangers qui se verraient facilement octroyer la nationalité ivoirienne, il accorda des droits politiques, comme le droit de participer à l'élection présidentielle, aux étrangers ouest africains. Encore davantage, il fit venir des étrangers, notamment des Burkinabé, qui furent installés à l'Ouest de la Côte d'Ivoire dans des villages créés pour eux. Et pour faciliter leur intégration, il a fait application d'une loi non promulguée de

41 Convention entre le Gouvernement de la Côte d'Ivoire et le Gouvernement de la République française relative à la circulation des personnes, Décret n 77-358 du 9 juin 1977 portant ratification de la Convention conclue entre la République de Côte d'Ivoire et la République Française, JORCl, du 4 juillet 1977, p. 1223.

42 Convention de sécurité sociale entre le gouvernement de la République de Côte d'lvoire et le gouvernement de la République française, JORCl, $\mathrm{n}^{\circ} 2$, du jeudi 12 janvier, 1995, p. 30.

43 F. Cooper, «Empire, nation et citoyenneté : la France et l'Afrique », XXXIII Conférence Marc-Bloch, op. cit. : https://www.ehess.fr/sites/default/files/pagedebase/.../2011 conference marc bloch.pdf, p. 8.

44 Ibidem.

45 CSCJ, arrêt $n^{\circ} 77$ du 23 janvier 1997 relative à un salarié d'origine béninoise qui avait conclu un contrat de travail en 1991 avec le Collège moderne Pascal Gueu (Man) en tant que professeur de français.

46 F. Houphouët-Boigny, Conférence de presse du Président de la République, Président du PDCl RDA, op. cit., p. 2: " Nous avons eu dans nos gouvernements des ministres venus de pays africains amis mais étrangers. Nous avons des préfets étrangers. Nous avons des ambassadeurs étrangers. Nous avons des chefs de services étrangers. Et nous avons dans la vie quotidienne, des paysans originaires d'autres territoires africains qui, avec les mêmes droits que les ivoiriens, participent à la construction nationale». 
1963 et d'une décision du bureau politique du PDCI RDA ${ }^{47}$, parti unique au pouvoir, selon lesquelles la terre appartient à celui qui la met en valeur ${ }^{48}$.

Pour certains observateurs, le miracle économique ivoirien des années 1970 tire sa source de cette politique d'ouverture. Mais ce regard bienveillant vis-à-vis de l'étranger a été remis en cause. L'égalité de traitement de l'étranger fait progressivement place à une inégalité de traitement.

\section{II - L'INÉGALITÉ DE TRAITEMENT DE L'ÉTRANGER}

L'inégalité de traitement de l'étranger en droit ivoirien concerne aussi bien l'étranger candidat à l'emploi (A) que le titulaire d'emploi face au licenciement (B).

\section{A - L'INÉGALITÉ DE TRAITEMENT DE L'ÉTRANGER À LA RECHERCHE D'EMPLOI}

Jusqu'en 1977, date de la création du Ministère du travail et de l'ivoirisation des cadres, la seule différence de traitement de l'étranger dans l'accès à l'emploi était le visa de travail ${ }^{49}$. Mais cette disposition était plus symbolique que réelle car, au regard du climat favorable à l'emploi des étrangers et des précautions prises pour encadrer sa délivrance ${ }^{50}$, l'administration du travail répondait quasi-systématiquement de façon favorable aux demandes de délivrance des visas. En outre, l'administration n'effectuait aucun contrôle en la matière. Ce qui fait que tout étranger pouvait conclure un contrat de travail sans avoir satisfait à cette formalité.

L'inégalité de traitement est née avec la création en 1977 du Ministère du travail et de l'ivoirisation des cadres, dont certaines circulaires ont instauré des plans d'ivoirisation limitant le recrutement des étrangers dans l'enseignement et dans les entreprises publiques. C'est à cette époque que le contentieux des visas de travail jusque-là latent a fait surface,

47 Y. Tano, "La précarité des titres fonciers en Côte d'Ivoire », in Pouvoirs publics et développement en Afrique, Toulouse, SEDUSS, 1992, p. 158: "Il y a suffisamment de terres cultivables pour tous; ce sont les bras qui manquent. Le Gouvernement et le Parti ont donc décidé, dans l'intérêt du pays, de reconnaître à tout citoyen d'origine ou d'adoption, qui met une parcelle de cette terre en valeur, quelle qu'en soit l'étendue, le droit de jouissance à titre définitif et transmissibles à ses héritiers ».

48 Loi non promulguée du 20 mars 1963. Pour plus de précision sur cette loi, voir S. Boni, op. cit., p. 156 ; M. Kone, Quelles lois pour résoudre les problèmes liés au foncier en Côte d'lvoire, Grain de sel, $n^{\circ} 36$, septembre-novembre 2006, p. 1.

49 Le visa est régi actuellement par l'article 7 du décret n 96-287 du 3 avril 1996 relatif au contrat de travail. Depuis l'indépendance, il a été régi successivement par l'article 1 D 3 alinéa 2 et 3 de la loi n 64-290 du 1er août portant Code du travail, JORCl, n 44, Spécial, p. 1059: « Il (le contrat de travail) est soumis au visa de l'OMOCl du lieu de l'embauchage et à défaut, aux visas de l'inspecteur du travail et des lois sociales ou de son représentant. En ce qui concerne les travailleurs embauchés hors de la République, le contrat de travail est soumis sur pièces au visa de l'OMOCl ».

50 CSCJ, 20 novembre 2014, arrêt $n^{\circ}$ 596/14, inédit. Le visa de cet arrêt est ainsi formulé: «Si l'autorité compétente pour accorder le visa n'a pas fait connaitre sa décision dans les quinze jours qui suivent le dépôt de la demande de visa, ce visa est réputé avoir été accordé », contrairement à la théorie du rejet implicite de la demande. 
permettant ainsi au juge de prononcer la sanction de nullité du contrat en l'absence de visa ${ }^{51}$.

Dès 1997, les mesures restrictives de l'accès à l'emploi de l'étranger ont été renforcées. Non seulement «l'ivoirisation des emplois» introduite dans les années 1977 a été étendue aux entreprises privées ${ }^{52}$, mais en outre, "préalablement à son engagement dans une entreprise établie en Côte d'Ivoire, tout travailleur non ivoirien doit être titulaire d'un contrat de travail ou d'une lettre d'embauche visée par le Ministère chargé de l'emploi sur un formulaire établi à cet effet ». Il en est de même des conditions d'obtention du visa qui ont été durcies ${ }^{53}$. Dans le même ordre d'idées, l'administration du travail est invitée à apprécier les demandes de visa en tenant compte de la situation de l'emploi dans le secteur concerné ${ }^{54}$. Des infractions et sanctions afférentes sont prévues à l'encontre des contrevenants, salariés ${ }^{55}$ et employeurs ${ }^{56}$.

Alors que l'on s'attendait à ce que la réforme du Code du travail de 2015 rectifie le tir, elle a abondé dans le même sens et érigé dans le Code du travail des dispositions considérées jusque-là comme simplement réglementaires ${ }^{57}$. Mais l'inégalité de traitement ne se limite pas à l'accès à l'emploi. Elle concerne également l'étranger titulaire d'un emploi.

\section{B - L'INÉGALITÉ DE TRAITEMENT DE L'ÉTRANGER TITULAIRE D'EMPLOI}

L'inégalité de traitement des étrangers titulaires de contrats de travail se traduit par la prise en considération de la nationalité comme motif de licenciement. II est question de discrimination puisque l'inégalité visée repose sur une raison que la loi n’autorise pas. Ce fut

51 CSCJ, 20 novembre 2014, op. cit. : «Attendu qu'il n'est point justifié que l'Office de la main-d'œuvre de Côte d'Ivoire a été effectivement saisi d'une demande visa concernant les époux Guillot, que la nullité qui s'en déduit, en application du paragraphe 8 de l'article 3 du décret n $65-121$ du 2 avril 1965 fixant les modalités d'établissement du contrat de travail, ouvre droit à des dommages et intérêts à la charge de l'ex-employeur »; voir également CSCJ, 15 janvier 1995, arrêt n05-91, inédit: "Mais attendu que les disposition ID3 du Code du travail qui prévoit la nullité du contrat en l'absence de visa de l'OMOCl sont d'ordre public ».

52 L'arrêté interministériel de 2004 précise clairement: «Toute vacance de poste doit faire l'objet de publication pendant deux mois auprès des organismes de placement agréés par l'État ». Voir également l'article 7 de cet arrêté qui instaure une différence de frais de visa selon que le candidat à l'emploi est d'origine ouest africaine (CEDEAO), africaine ou non africaine, et selon qu'il s'agit de l'occupation d'un poste de direction.

53 Art. 2 alinéa 2 de l'arrêté : «Si au terme de cette période d'un (1) mois, le poste n'est pas pourvu par un national, l'employeur est autorisé à recruter tout candidat répondant au profil requis dans le strict respect des dispositions du présent arrêté "».

54 Art. 4 alinéa $1^{\text {er }}$ de l'arrêté : «Toute demande de visa de travail ou de renouvellement de ce visa est examinée au regard de la situation de l'emploi dans le secteur concerné ».

55 Le non-respect par l'étranger candidat à l'emploi des dispositions réglementaires sur l'emploi des étrangers en Côte d'lvoire est frappé par l'interdiction de travailler en Côte d'Ivoire.

56 En plus du paiement des frais de visa, l'employeur est sanctionné par une pénalité égale à trois mois de salaire mensuel brut du travailleur par année d'infraction ». Voir art. 10 de l'arrêté.

57 Art. 11.1, alinéas 2 et 3: «Toute vacance de poste doit faire l'objet de déclaration auprès de l'organisme public de placement, de publication dans un quotidien national à grand tirage et éventuellement dans tout autre moyen de communication. Si au terme d'une période d'un mois à compter de la première publication, aucun national n'a satisfait au profil requis, l'employeur est autorisé à recruter tout autre candidat». 
le cas, par exemple, dans un arrêt rendu le 7 mai 2005 par la Cour d'appel d'Abidjan. Dans cet arrêt, non seulement l'AGEPE ${ }^{58}$ a répondu négativement à la demande de l'employeur tendant à renouveler le contrat de travail, mais elle a demandé à ce dernier "d'ivoiriser» le poste ${ }^{59}$. Comme dans le cas précédent, l'inégalité de traitement des étrangers titulaires d'emploi à sa source de la politique « d'ivoirisation des emplois » mise en œuvre dans les années 1970, à l'instar de la majorité des pays d'Afrique francophone tels le Sénégal, le Gabon ou encore le Cameroun ${ }^{60}$ qui avaient décidé de s'affranchir de la mainmise française sur l'encadrement national.

En Côte d'Ivoire par exemple, jusqu'en 1970, la majorité des cadres supérieurs de la fonction publique étaient de nationalité française. L'objectif affiché était donc de favoriser le remplacement de ces derniers par des cadres locaux, d'où l'obligation pesant sur toutes les entreprises publiques de présenter des plans d'ivoirisation de leurs personnels.

Le contentieux de l'ivoirisation des emplois qui s'en est suivi a donné lieu à une controverse judiciaire. La question était posée au juge de savoir si l'ivoirisation des emplois pouvait être considérée comme un motif légitime de licenciement? Pour la majorité des juges du fond, la réponse était affirmative ${ }^{61}$. S'il est exact que la Cour suprême n'était pas exempte de critique, certains de ses arrêts abondent dans le sens des juges du fond et, dans la plupart d'entre eux, elle a reconnu la discrimination dont était victimes les salariés concernés ${ }^{62}$.

Mais faute de traçabilité de la jurisprudence en Côte d'Ivoire, cette décision qui devait servir de guide d'interprétation des textes pour les juges du fond n'a pu déployer tous ses effets pédagogiques: plusieurs décisions statuant en sens contraire furent ainsi repérées ultérieurement ${ }^{63}$.

58 Agence d'Étude et de Promotion de l'Emploi.

59 Voir par exemple le courrier de l'Agence d'Études et de Promotion de l'Emploi (AGEPE), en réponse à une demande de la société SAECI-ASTRAL formulée le 24 octobre 1996 visant à renouveler le contrat de travail de son salarié d'origine étrangère. Dans ce courrier, selon l'arrêt qui l'évoque, non seulement l'AGEPE a refusé de viser le contrat de travail qui a lui a été soumis par l'employeur, mais a adressé un courrier à ce dernier pour demander d'ivoiriser le poste litigieux. Voir Abidjan, $1^{1}$ ére Chambre sociale, arrêt $n^{\circ} 503$ du 7 mai 2005, Juris social, nº 65, p. 24.

60 I. L. Miendjiem, «Egalité et discrimination en droit camerounais du travail », thèse pour le doctorat du $3^{\text {ème }}$ cycle, Université de Yaoundé II, 1995-1996.

61 Abidjan, 1 ère Chambre sociale, arrêt n ${ }^{\circ} 503$ du 7 mai 2005, op. cit., p. 23 : Dans cette décision, le juge a déclaré illégitime le licenciement d'un travailleur de nationalité étrangère non pas parce que le motif invoqué - la nationalité étrangère - ne pouvait pas servir de fondement à la rupture du contrat, mais par carence d'ivoirisation du poste; Daloa, 24 juin 1998, arrêt n 129, Juris-social, $n^{\circ} 13$, janvier 2002, p. 12: Cette juridiction a estimé que l'ivoirisation de l'emploi d'un professeur de collège à la suite des circulaires du Ministre de l'Éducation nationale, autorité de tutelle de l'employeur, constitue un motif sérieux et légitime de licenciement.

62 CSCJ, formation sociale, 20 mars 1997, arrêt $n^{\circ}$ 75, Juris-social, $n^{\circ}$ 2, février 2002, p. 6: « Constitue un abus dans le licenciement opéré, un employeur qui, pour cause d'ivoirisation des postes contenue dans les instructions reçues du Ministre de la Fonction publique, a licencié des travailleurs d'origine étrangère à qui il n'est reproché aucune faute professionnelle ».

63 CSCJ, 22 mai 2014, arrêt n³ 360/14 inédit, op. cit. 


\section{Migrations INIERNATIONALES Et TRAVALL EN Côte d'IVOIRE}

Pour conclure, il ressort de l'analyse ci-avant développée que la Côte d'Ivoire, en tant que pays d'accueil et donc d'immigration, a d'abord fait de l'assimilation de l'étranger une réalité tangible. Mais très vite, le pays a été rattrapé par la crise économique et son corolaire, la rareté de l'emploi qui, comme partout ailleurs, a accusé l'étranger d'en être en à l'origine. En ont suivi des mesures restrictives prenant parfois l'allure de véritables discriminations fondées sur la nationalité.

\section{NANGA SILUÉ}

Professeur agrégé de droit privé et sciences criminelles, Doyen de I'UFR SJAG de I'Université Alassane Ouattara, Bouaké-Côte d'lvoire

Thématiques de recherche: droit social, droits fondamentaux, sources du droit, droit comparé

\section{Publications:}

N. Silué, " Egalité entre I'homme et la femme dans la famille ivoirienne ou le droit hypocrite ", Mélanges A. Lyon-Caen, Dalloz, 2018.

N. Silué, Dialogue social en Côte d'lvoire : de l'étouffement à la libération du droit, in Dialogue social dans les pays de l'espace OHADA et ailleurs dans le monde. La part du droit, L'Harmattan, 2018. 